\title{
Chapitre 3 Deux statuts pour le dire autre représenté : comme objet et comme source du Dire
}

Les deux actes d'énonciation, A et $\boldsymbol{a}$, que - définitoirement - met en jeu tout fait de RDA s'y articulent de deux façons différentes : relativement au Dire en cours, le discours autre auquel il réfère n’y est pas représenté avec le même statut.

Dans l'une, qui correspond à l'ensemble des modes traditionnellement répertoriés comme « discours rapporté », DD, DI, DIL, le discours autre est, précisément, cela même que « rapporte » l'énoncé $\mathbf{E}$, y occupant la place, centrale, de l'objet du dire - de ce dont il parle :

(1) Jean dit qu'il va bien. Jean a dit : « Je vais bien ». Jean les a rassurés : « il va bien ».

Dans l'autre, c'est « de biais » que le discours autre peut intervenir dans l'énoncé, convoqué au titre de source du dire - discours à partir duquel il se fait :

(2) Jean va bien, d'après Paul. Jean va « pas mal du tout » pour reprendre les mots de Paul.

En circonscrivant, dans le champ du métadiscours, le champ de la RDA, on a déjà rencontré (chap. 1), comme partie prenante de celui-ci, ce qui dans les mouvements de retour du dire sur lui-même - méta-énonciation explicite ou modalisation - « passait par » la représentation d'un discours autre.

Au-delà de ce constat, il importe de revenir sur ce deuxième type de mise en jeu du discours autre comme modalisation du Dire, d'y reconnaître cette « Modalisation par Discours Autre » (MDA) pour l'un des deux versants sur lesquels se réalise la $\mathrm{RDA}^{1}$. De ce champ, très vaste, dont certains secteurs - ceux, notamment, où le discours autre est représenté comme la source d'un élément du Dire ont été l'objet d'études approfondies, il ne s'agit pas ici de viser une description détaillée de ses multiples formes et de leur fonctionnement, mais d'en proposer, schématiquement, une « cartographie » assurant la place, au sein de la RDA, de son versant modalisant, à côté, différentiellement, de la trilogie DD, DI, DIL affectée à la prédication d'un fait de discours autre.

On envisagera le champ de la MDA à travers deux oppositions. L'une qui, interne, le divise en fonction du plan du Dire auquel est incident le discours autre convoqué : celui du contenu (1) ou celui de la forme (2). L’autre qui, par rapport à son extérieur, le délimite en fonction du mode sur lequel le discours

1 Je reprends ici l'une des oppositions proposées comme structurant le champ de la RDA dans Authier-Revuz (1993a : 39), (1997), (2004a).

ว Open Access. () 2020 Jacqueline Authier-Revuz, published by De Gruyter. (cc)BY-NC-ND This work is licensed under the Creative Commons Attribution-NonCommercial-NoDerivatives 4.0 License. https://doi.org/10.1515/9783110641226-008 
autre « affecte » l'énoncé : celui, illustré dans l'exemple (2) modalisant au sens strict, d'un renvoi à une source distincte de l'énonciateur $\mathbf{L}$ du Dire, de sa prise en charge d'un élément de ce dire - assertion, modalisée comme seconde (MAS, 1.1), ou manière de dire, modalisée, autonymiquement, comme empruntée (MAE, 2.1) - ; ou celui, plus « lâche » du Dire énoncé explicitement - contenu (1.2) ou matérialité des mots (2.2) - comme « en rapport » avec, accompagné par un discours autre « conjoint », comme en :

(3) Contrairement à ce qui se dit, Jean va bien. Jean va bien (il dit même « super bien).

\section{Discours autre incident au plan du contenu du Dire}

\subsection{La modalisation de l'assertion comme seconde (MAS)}

\subsubsection{Une opposition sémantique, énonciative, syntaxique entre DA objet ou source}

Si on compare l'énoncé (4), « mondain » aux énoncés (5a et 5b) relevant de la RDA

(4) Marie a fait une longue promenade.

(5) (a) Jean dit que Marie a fait une longue promenade.

(b) Marie a, d'après Jean, fait une longue promenade.

on voit que, par rapport à (4) qui est une assertion portant sur un événement quelconque - non langagier - du monde, la promenade de Marie, les énoncés (5) diffèrent :

- (a) est une assertion (de $\mathbf{L}$ ) portant sur un autre événement : celui, de type langagier, que constitue le dire de Jean (au sujet de la promenade de Marie)

- (b) est une assertion de $\mathbf{L}$ portant sur le même événement que (4) - la promenade de Marie - commentée, modalisée comme provenant d'un ailleurs langagier - le dire de Jean.

( $a$ et $b$ ) ont certes la même valeur de vérité, mais ils s'opposent par une répartition-hiérarchisation différente des contenus au plan sémantico-énonciatif :

- (a) parle d'un dire (qui parle du monde)

- (b) parle du monde (d'après un dire).

Dans la structuration (a), le dire autre apparaît comme l'objet de la prédication de $\mathbf{L}$, ce sur quoi elle porte et sur quoi il s'engage ; mais assertant que $\mathbf{l}$ dit que $P$, 
le fait du dire autre, il n'asserte pas $\mathrm{P}$ (le fait que Marie ait fait une longue promenade), le contenu de ce dire autre ${ }^{2}$.

Dans la structuration (b), en revanche, $P$, d'après le dire de l, le contenu P (la promenade de Marie) est bien asserté par $\mathbf{L}$, cette assertion étant secondairement, additionnellement, caractérisée comme issue - reçue, empruntée, extraite... d'un dire autre, premier ; et celui-ci reçoit, dans le dire, le statut d'élément participant, au titre de source, à la modalisation de l'assertion.

Dans un récit comme :

(6) Jean est arrivé en retard, il a dit qu'il était pressé et il est reparti aussitôt.

trois événements, l'arrivée de Jean, son acte de parole, son départ, constituent respectivement les référents de trois propositions. Si l'on ajoute, en tel ou tel point de la phrase, l'élément modalisateur d'après $\boldsymbol{l}$ :

(7) (D’après Marie) Jean est arrivé en retard, (d'après Marie) il a dit qu'il était pressé et (d'après Marie) il est reparti aussitôt.

on voit que « ce dont parle » l'énonciateur, ce qui est l'objet de son dire, ne change pas, mais que telle de ses assertions au sujet du même référent est donnée comme

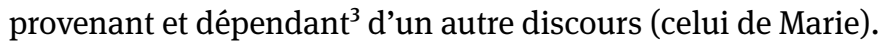

On distinguera les deux statuts conférés en RDA au discours autre représenté : (a) objet du Dire, par les formes (DD, DI, Bivocal-DIL) de « prédication d'un discours autre » (PDA), vs (b) source du Dire par les formes relevant de la « modalisation par discours autre » (MDA). La RDA est, pour le premier, constitutive du dire « $\boldsymbol{l}$ dit $\mathrm{P}$ », là où, pour le second, elle intervient dans le dire de $\mathrm{P}$ au titre d'une modalisation passant par une RDA ( $c f$. ci-dessus chap. 1.2.4).

La différence d'angle sous lequel un même fait de discours $\boldsymbol{a}$ est représenté dans un énoncé A selon qu'il y est parlé de ou d'après lui, apparaît dans le caractère inégalement naturel selon lequel les énoncés (a) ou (b) peuvent se combiner

2 Cf., par exemple, Martin (1983/1992 : 107), posant que, dans le DI, l dit que $P$, « la valeur de vérité de "P" n'est pas prise en charge par L (la responsabilité est laissée à l) », ou Recanati (1981 : 219) : « Dans un exemple de discours rapporté comme « Pierre dit : "Henry est généreux" » ou "Pierre dit que Henry est généreux", ce que dit le locuteur ne se confond pas avec ce que dit celui dont il rapporte les propos. La parole du locuteur a pour objet la parole d'un autre et s'en distingue : le locuteur ne dit pas que Henry est généreux, il dit que Pierre le dit [...] ». 3 Cette « dépendance » à un autre dire dans laquelle un Dire inscrit une assertion est formulée ainsi par Van Raemdonck (2002 : 173) : « Dans cette tournure [à ce qu'elle me dit/selon elle], l'énonciateur assume son énoncé dans le cadre défini par les compléments adverbiaux. Il prend donc en charge l'élément [... Pierre arrivera ...] pour autant qu'il soit ramené à la personne que l'on « cite » $[. .]$.$» .$ 
- en réponse ou en enchaînement par coordination - à des énoncés référant à des événements ${ }^{4}$ :

(8) Qu'est-ce qu'il fait, Jean ?

(a) Il dit que la subvention est annulée.

(b) ? D’après lui, la subvention est annulée.

(9) (a) Jean a dit que Sarah reprenait le rôle, et il est parti.

(b) ? D’après Jean, Sarah reprend le rôle, et il est parti.

À la différence de statut entre (a) et (b) répond la différence de contraintes spécifiques sur les formules de la représentation d'un DA objet ou source du dire.

Schématiquement, on peut opposer, au caractère libre de la modalité d'énonciation du DA représenté comme objet,

(10) 1 a dit que $\mathrm{P}$; demandé si $\mathrm{P}$; ordonné que $\mathrm{P}$

l a dit « Il vient »; «Vient-il ? »; «Viens !»

sa restriction à un contenu assertif dans le cas du DA représenté comme source via les selon $\mathbf{l}$, à entendre $\mathbf{l}$, d'après ce qu'il dit, il paraît etc. ; ainsi, l'injonction est-elle exclue (11), et si l'interrogation est possible (12), ce n'est pas le questionnement qui est donné comme modalisé par un emprunt (à Jean), mais les assertions alternatives ouvertes par celui-ci (il va/ne va pas faire beau) :

(11) *Viens! selon Jean.

(12) Va-t-il faire beau, selon Jean?

Remarque 1 : Sur la restriction à l'assertion de la MAS. Cela, bien entendu, ne signifie pas que l'énoncé auquel réfère la MDA comme sa source ait été assertif, mais que l'assertion qu'il formule puisse en être extraite : ainsi, «Selon Jean, l'air est de mauvaise qualité » peut-elle provenir (entre autres...) de «Quittez au plus vite cette banlieue polluée ! ». Par ailleurs, il ne faut pas confondre cette assertion prélevée comme contenue dans l'énoncé non nécessairement assertif, avec la possibilité d'énoncés tels que : «D'après les questions/les ordres de Jean, $\mathrm{P}$ » où $\mathrm{P}$ n'est pas une assertion empruntée (MDA) au dire de Jean, mais inférée à partir de celui-ci, $c f$. par exemple :

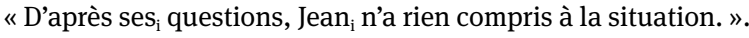

On notera que le caractère exclusivement assertif de ce qui est considéré comme second interdit les assimilations parfois envisagées ( $c f$. chap. 4 note 68 ) de selon $l, P$, ou plus généralement, de la MAS, avec le DIL qui, lui, se déploie sans restriction sur tout le clavier illocutoire :

(13) Jean peste : on ne l'a pas attendu. / Se moque-t-on de lui ? / Qu'on vienne le chercher, parce que lui, il ne fera pas un pas de plus!

$4 C f$. les observations de D. Coltier (2002) sur les différences de fonctionnement entre selon $A$ et A V dire. 
On peut de même opposer à l'indifférence de la configuration (a) de PDA (prédication d'un discours autre) quant au caractère effectif du DA représenté :

(14) il a dit, il n'a pas dit, il dira, il dirait, a-t-il dit...

la contrainte pour la MDA (b) que l'assertion « source » dont le dire se représente comme issu, soit réalisée, antérieurement à celui-ci ${ }^{5}$ :

(15) Selon ce que $\boldsymbol{l}$ a dit $v s$ * selon ce que $\boldsymbol{l}$ n'a pas dit, dira peut-être, aurait pu dire, dirait si,... ${ }^{6}$

Enfin à l'opposition entre les statuts des discours autres objet vs source du dire, répond, au plan du fonctionnement syntaxique, l'opposition entre les contraintes de la syntaxe « centrale », phrastique, des premiers ( $\boldsymbol{l}$ dit $P, \boldsymbol{l}$ dit que $P, \ldots$, et la liberté propre à la « périphérie » des circonstants extra-phrastiques des seconds $(P$, selon $\boldsymbol{l}, . .$.$) qui, différentiellement, apparaissent, en effet, comme :$

- facultatifs (suppressibles) et, de ce fait, détachables, intonativement ou typographiquement (par parenthèses, doubles tirets, placement en note, ...) :

(16) a La victime est (selon le témoin principal) rentrée à l'heure usuelle.

b La victime est rentrée à l'heure usuelle'.

1. selon le témoin principal.

- mobiles, en cas d'incidence globale :

(17) [@=selon le témoin principal] @ La victime @ est @ rentrée @ à l’heure usuelle @.

- $\quad$ aptes à des incidences partielles et multipliables au sein d'une même phrase :

$(18)^{7}$ Il a discuté, âprement selon Jean, le contrat, jusque, d'après Marie, dans les moindres détails.

5 Cf. Charolles (1987 : 254) : « dans “selon A, p”, $p$ doit avoir fait l'objet d'une énonciation quelconque de la part de $A$ » (idt). Ce renvoi obligatoire à un $\mathbf{a}^{0}$ antérieur d'où est « extraite » l'assertion modalisée n'implique ni l'identité, ni même une synonymie de type linguistique, entre formulation « origine » et assertion seconde (le rapport paraphrastique « en substance » y est possible, $c f$. chap. 7.2.1.2), ni que le discours antérieur ait été " extériorisé » : certaines formes de MAS - selon l, pour $l$, ... mais pas, bien sûr, à l'entendre, à l'en croire, ... - sont susceptibles de renvoyer à un discours intérieur prêté par $\mathbf{L}$ à $\mathbf{l}$ sur une base non-exclusivement discursive mais faite aussi d'attitudes, de comportements ... (voir les remarques de D. Coltier (2002), concernant selon l). 6 Bien entendu, de tels syntagmes sont possibles, mais avec des valeurs autres que celles de la MDA : par exemple dans "selon ce qu'il dira, on ira ou non ", selon introduit un circonstant intra-phrastique à valeur de « en fonction, conformément ». $C f$. notamment Coltier $(2000,2002)$. 7 Comparer à l'extraction d'un constituant par $\mathbf{l}$ dit que c'est... que : « Jean dit que c'est âprement qu’il... / Marie dit que c'est dans les moindres détails que... » qui n’est pas réitérable. 
Apparaît donc, ici, distincte des formes du DA-objet du dire, au plan sémantique, énonciatif, syntaxique, une configuration de MDA opérant au plan de l'assertion du Dire, donnée comme seconde par rapport au DA-source dont elle est issue, et qui sera désignée comme "modalisation (du dire) en/comme assertion seconde » $(\mathrm{MAS})^{8}$.

La question de la référence explicite à l'acte d'énonciation autre a partage aussi les deux versants - discours autre objet vs source - de la RDA : élément clairement nécessaire du premier, qui « parle d'un dire », il n'en va pas de même sur le second versant où l'on " parle... d'après un dire » et où, selon les formes de MAS, la question du renvoi à l'acte d'énonciation autre reçoit des réponses diverses, comme le montrent, par exemple, l'opposition, sur ce plan, entre :

(19) a) Il paraît que $P$.

b) À ce qu'on dit depuis quelques jours, $\mathrm{P}^{9}$.

On note en revanche que, si une partie des formes modalisantes implique un acte d'énonciation sans y référer explicitement, que ce soit avec désignation d'un $\boldsymbol{l}$ (selon, d'après, pour, suivant l) ou sans (conditionnel d'ouï-dire, il paraît, censément...), ce n'est pas là une propriété nécessaire de la MAS, qui est apte à référer - avec un autre statut que celui d'objet du Dire - à l'acte d'énonciation source, et avec la plus grande précision :

(20) D'après ce qu'a dit X, l'autre jour, quand Y l'a interviewé au 20 heures sur TF1, la centrale n'est pas dangereuse.

Selon la déclaration faite par X à l'issue de la rencontre avec les représentants du personnel, aucun accord n’a été trouvé.

Remarque 2 : MAS et Référence à l'acte d'énonciation source. C'est la question à laquelle, à propos du « conditionnel “journalistique” d'information empruntée », s'attache Kronning (2002), soucieux - pertinemment - de le distinguer du « discours rapporté » (DD, DI). On peut noter que les deux observations qu'il formule, valides toutes deux pour le conditionnel, ne peuvent pas être étendues à l'ensemble de la MAS : si pour la MAS, comme pour le conditionnel,

« l'entité empruntée [...] n’est pas un acte d'énonciation (avec son contenu épistémique) mais une proposition assertée attribuée à autrui » (Kronning 2002 : 572),

il n'en va pas de même de l'observation selon laquelle

8 Présent, en français, dans d'après, ou suivant, le caractère « second » de l'assertion par rapport à sa source, s'affiche dans le secondo italien ou le segundo espagnol (et portugais).

9 Forme chère à La Fontaine : « Tous les gens querelleurs, Jusqu'aux simples mâtins / Au dire de chacun, étaient de petits saints. » (Fables VII-1) ; «Elles avaient la gloire/ de compter dans leur race (à ce que dit l'Histoire) / L'une certaine chèvre au mérite sans pair [...] » (Fables XII-4). 
[le contenu que] « l'instance médiatisante a extrait d'un acte d'énonciation préalable [est transmis] sans faire référence à cet acte (ces actes) et sa (leurs) localisation(s) temporelle(s) (ibid. : 570).

Si, en effet, dans l'ensemble des formes de MAS, qui toutes impliquent un acte d'énonciation autre, certaines - comme le conditionnel d'ouï-dire - le font sans-référer explicitement à celui-ci, ce n'est pas là une propriété caractéristique de la MAS qui - avec un autre statut que celui d'objet du Dire - peut référer explicitement à l'acte d'énonciation source. La possibilité en MAS, de faire ou non explicitement référence à l'acte d'énonciation placé en position de source, opère une différenciation à l'intérieur du mode entre les formes : en rendre compte supposerait une description distributionnelle fine de l'ensemble - vaste - des formes de MAS.

De façon schématique ${ }^{10}$ on peut évoquer plusieurs sous-ensembles :

- celui des formes excluant l'explicitation (en « dire ») de l'acte d'énonciation source, soit strictement :

il paraît ; à ce qu’il paraît ; conditionnel ; officiellement ; de notoriété publique ; censément ; prétendument...

soit admettant la référence à l'énonciateur $\boldsymbol{l}$ :

pour l (vs * pour ce que dit $\boldsymbol{l}$ ) ;

- celui des formes requérant cette référence explicite :

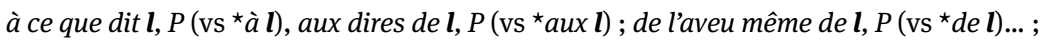

- celui des formes permettant, de façon variée, d'expliciter l'acte source, le discours ou le $\mathbf{l}$ :

selon $1 . . .$, selon ses dires, selon la déclaration de là l'issue de la rencontre..., selon des sources concordantes... ;

d'après $\mathbf{l}$, d'après le bulletin météo, le témoignage de $\mathbf{l}$, d'après une source officieuse, d'après les manuels qui font référence, d'après la rumeur, d'après ce qu’il a déclaré publiquement...

- celui des formes passant par l'explicitation de la réception, par $\mathbf{L}$, de l'acte de dire d'un $\boldsymbol{l}$ (explicite ou non) :

à en croire $\mathbf{l}$, la rumeur, les experts ; à suivre $\mathbf{l}$; si l'on en croit, si on se fie à ce que dit $\boldsymbol{l}$; si l'on s'en tient à ce qu'en dit $\boldsymbol{l}$; à vous entendre, tout était normal ; à lire sa déclaration, il n'était au courant de rien...

\subsubsection{Approches diverses de la MAS}

\section{(i) La MAS sous l'angle évidentiel/médiatif}

Relevant pleinement de la RDA, c'est cependant dans d'autres cadres que cette configuration a été le plus étudiée ${ }^{11}$-, comme partie prenante du fonctionnement

10 C'est-à-dire, notamment, sans distinguer formes univoques de MAS et formes ambiguës (ex. : d'après $l, P$ vs d'après ce que $\mathbf{l}$ a dit, $P$, qui peut aussi signifier d'après ce qu'il a dit, je peux conclure $P)$; ni sans préciser la différence d'explicitation de la source, entre nom de parole ou verbe de dire (ex. : d'après son témoignage, d'après ce qu'il a dit).

11 Notamment, le conditionnel de MAS - «d'altérité énonciative », « d'allusion au discours de l'autre » (Haillet 1995, 2002), « d'information incertaine » (Dendale 1993), « conditionnel média- 
de « l'évidentiel »/ « médiatif », ou de la problématique de la « prise en charge », et en rapport avec le champ de la modalité épistémique.

Ainsi, dans le cadre « évidentiel/médiatif » du marquage des «sources du savoir », c'est-à-dire de la façon dont l'information a été obtenue par l'énonciateur, les présentations et mises au point de P. Dendale et L. Tasmowski (1994 et 2001) ou de Z. Guentcheva (1996, 2004, par exemple) fournissent un balisage de ce champ, dont on retiendra :

- au plan historico-bibliographique, que, au-delà des jalons que constituent l'apparition (chez Boas en 1911, chez Sapir en 1921) de la problématique distinguant les « savoirs » obtenus par expérience directe (perception), ouï-dire ou inférence, et celle des termes d'évidentialité (chez Boas en 1947 ${ }^{12}$, chez Jakobson en 1957 dans le célèbre article sur les "shifters » ${ }^{13}$ ), et de médiatif (G. Lazard 1956), c'est à partir des années 80 que se développe l'exploration systématique du champ, avec une abondante bibliographie dans laquelle, outre les références déjà citées, on notera le travail « repère » de Chafe et Nichols (1986) ;

- au plan terminologique, que l'alternative « évidentialité/médiatif » correspond, d'une part, au refus, notamment chez Guentcheva ${ }^{14}$, de l'importation en français du faux-ami " evidence », qui, en anglais, renvoie aux moyens de justification, de preuve... pour ce qui précisément, n'est pas « évident ${ }^{15}$, et, d'autre part, à un strict ancrage du « médiatif » dans les formes de langue, là où l'« évidentialité » relève d'une catégorie " cognitive » ;

- au plan conceptuel, enfin, que l'une des questions récurrentes est celle - complexe, et tranchée selon des modes divers suivant les auteurs - du rapport à poser entre évidentialité, modalisation, et modalité épistémique.

tif » (Kronning 2002), par exemple - ou certains adverbiaux « énonciatifs », selon, d'après, pour l (Charolles 1987, Coltier 2000, Borillo 2005, Schrepfer-André 2004...).

12 Dans une grammaire parue de façon posthume, $c f$. Jacobsen (in Chafe et Nichols 1986 : 3-28). $13 C f$. dans la traduction française (Jakobson 1963: 183) : « Nous proposons d'appeler testimonial (anglais evidential) la catégorie verbale qui fait entrer en ligne de compte [...] la source d'information alléguée relativement au procès de l'énoncé ».

14 "Le terme de "médiatif" n’est pas encore répertorié dans le corps commun des concepts linguistiques. Il a été retenu ici pour désigner une catégorie grammaticale qui au moyen de procédés grammaticaux [...] permet à l'énonciateur de signifier que l'information fournie à propos des faits énoncés n'est pas issue d'une connaissance directe des faits, mais d'une connaissance perçue de façon médiate. » (Guentcheva 1996).

$15 C f$. aussi le vif rejet par J. Rey-Debove (2003 : 339) de cet emprunt à l'anglais « qui ne veut rien dire en français ». 
De façon schématique, on opposera à l'énoncé non marqué

(21) il est là.

dont « le locuteur asserte [le] contenu sans préciser comment il a eu l'information », l'ensemble des indications concernant ce « comment » ; et c'est à l'intérieur d'une opposition tripartite entre
(22) (a) perception -
il est là, je l'ai vu.
(b) inférence -
il doit être là, les volets sont ouverts.
(c) emprunt -
il paraît qu'il est là.

que sera posé, différentiellement, ce qui, (c), correspond à notre MAS, c'est-àdire, pour une information, sa caractérisation comme : médiate, par recours à un discours autre source.

Née de l'étude de langues dans lesquelles cette dimension de marquage différentiel des types d'obtention d'une information est grammaticalisée ${ }^{16}$ dans un système d'oppositions morphologiques obligatoires, souvent attachées au verbe, cette problématique s'est étendue, comme catégorie sémantico-énonciative, fortement liée à la question de la modalité et de la prise en charge, aux langues qui, comme le français, ne présentent pas de « système », mais un ensemble de moyens disparates - adverbes, auxiliaires modaux, valeurs temporelles dites modales, parenthétiques, syntagmes circonstanciels... - susceptibles d'être décrits comme correspondant aux divers « types » d'obtention de savoir. Dans cette perspective, c'est dans son opposition (par exemple) au type " par inférence/supposition » (il doit/peut/paraît/semble être là ; sûrement, selon toute vraisemblance il est là) qu'est dégagé le type " par emprunt » appréhendant - depuis une autre perspective que celle de la « représentation du discours autre » - le même domaine de faits que la MAS (selon, d'après, pour l, il est là ; il est, paraît-il, là ; il serait là, à ce qu'on dit...).

L'intérêt, pour le champ de la RDA, des travaux relevant de la perspective évidentielle/médiative est double.

D'une part, c'est le même ensemble de phénomènes qui se trouve - à l'intersection des deux champs de la RDA et de l'évidentialité/médiatif - appréhendé à travers ces deux perspectives ; aussi les nombreux travaux portant sur les formes

16 À des degrés de complexité divers, depuis les oppositions classiquement relevées en turc, bulgare, arménien, géorgien, albanais.... jusqu'au fameux tuyuka ( $c f$. par exemple Dendale-Tasmowski 1994 : 4) - avec son paradigme de cinq morphèmes affectant un même contenu informatif, de l'indication qu'il a été obtenu par 1) la vue, 2) l'ouie, 3) des indices matériels, 4) le dire d'un autre, 5) une supposition. 
et le fonctionnement de la valeur spécifique d'emprunt (ouï-dire, « hearsay », rapporté, de « seconde main »,...) de la catégorisation évidentielle/médiative de l'information apportent-ils une contribution d'autant plus précieuse à la description de ce secteur de la RDA qu'est la MAS que la tradition des études consacrées au « discours rapporté » l'a sensiblement négligé au profit des formes (DD, DI, DIL) à DA objet.

D'autre part, c'est souvent dans le cadre des études inscrites dans le champ évidentiel/médiatif que le souci de définir nettement cette catégorie a conduit à dégager ce qui oppose, dans les faits relatifs au "discours autre ", ceux qui relèvent du médiatif/évidentiel (notre MAS) et ceux qui lui sont étrangers (classiques « discours rapportés » à DA-objet) ${ }^{17}$, contrairement au mode peu différenciateur sur lequel les formes de MAS se trouvent aisément « annexées » comme une marge à un « cœur » DD-DI-DIL, dans des études consacrées au « discours rapporté » ou des analyses de discours faisant intervenir ce champ. On revient sur ces points ci-dessous.

\section{(ii) La MAS - à reconnaître comme mode propre, à part entière, de la RDA.}

Contrairement à ce qu'il en est dans le champ évidentiel/médiatif qui fait largement place dans ses investigations à ce qui y relève de l'axe de « l'ouï-dire », la reconnaissance de la modalisation du dire en assertion seconde (MAS) comme relevant pleinement de la RDA, sur le mode différencié du DA représenté à titre de source (et non d'objet) du dire, est loin d'être aboutie dans le champ de ce qui relève de la « représentation d'un discours autre ».

$17 C f$. par exemple Kronning $(2002$; 570) dans une étude consacrée au « conditionnel médiatif » : notant que celui-ci « est de temps à autre censé relever du "discours rapporté” (Rosier 1999) ", et que des linguistes " assimilent le discours rapporté à la catégorie médiative ("évidentielle") de "l'emprunt" ou de la "citation" ", il oppose son point de vue, selon lequel « il est important de distinguer, pour des raisons tant linguistiques que conceptuelles », le discours rapporté « qui rend un acte d'énonciation, un dire, en le présentant comme tel - [DD ou DI] - de la médiation épistémique qui dans le cas de "l'information empruntée" transmet un contenu épistémique que l'instance médiatisante a extrait d'un acte d'énonciation préalable [...]. » $C f$. également Z. Guentcheva (1994 : 12), (2004 : 27) qui, à propos des langues à « marqueurs médiatifs » morphologiques, fait nettement apparaître qu'il est nécessaire de "soigneusement distinguer » ce qu'elle appelle « l'énonciation médiatisée », d'avec le fait, autre, du discours rapporté, et combien «l'étiquette "faits rapportés" peut induire en erreur et conduire à penser que cette valeur [d'ouï-dire] du MED [= médiatif] [...] est de même nature que le discours indirect (DI) », là où, au contraire - derrière leur commune indication de source énonciative autre pour un contenu - ce sont « deux statuts différents » qui apparaissent, dont l'assimilation relèverait d'« d'un rapprochement hâtif et approximatif». 
Alors que ses formes - d'une grande variété - sont d'un emploi constant dans les genres les plus divers (à l'oral comme à l'écrit ; dans les conversations familières, la presse, les écrits relevant de la "didacticité », de la critique, du débat d’idées, de la recherche, les récits historiques, mémoires, etc.) la MAS est très majoritairement absente des présentations contemporaines de la RDA dans :

- les manuels scolaires ${ }^{\mathbf{1 8}}$, en dépit de l'évidente nécessité pédagogique de faire accéder les « apprenants » à la maîtrise d'une alternance, si souvent pratiquée dans les discours, entre ces deux « abords » du discours autre- comme objet ou comme source $-{ }^{19}$;

- les ouvrages généraux - grammaires, dictionnaires - spécialisés, reconduisant ${ }^{20}$, aux chapitres ou articles « discours rapporté », l'inventaire traditionnellement restreint aux trois formes du premier type ; ainsi, par exemple, dans cette formulation ${ }^{21}$ :

Le terme de discours rapporté désigne les différentes modalités d'intégration d'un discours extérieur dans un premier discours. Les différentes formes en sont le discours direct, le discours indirect et le discours indirect libre (Arrivé et al. (1986:236))

où l'on peut noter que la généralité de la définition du « discours rapporté » - en termes « d'intégration » d'un discours dans un autre - répondrait pourtant, de

18 Par exemple, dans cet échantillon (que je dois à Catherine Boré) de manuels en usage : Grammaire 5e, Hatier, 2001, chap. 16 ; 4e, Magnard, 1998, chap. 14 ; 3e, Texto, 2003, leçon 24 ; 3e, Belin, 2003, chap. 8 , bornant le parcours des « moyens de rapporter des paroles » à la trilogie DD, DI, DIL (parfois associée au «narrativisé »).

19 Ce que souligne, précisément, M.-J. Reichler-Béguelin (1992 : 205) - dans une perspective pédagogique en français langue seconde - parmi les points à ne pas manquer parmi les ressources ou difficultés pour « exercer » les apprenants à la production, dans le champ de la restitution de discours autres : « Le DR entre d'autre part en concurrence avec des formulations modalisées : assumées par le locuteur de l'énoncé en cours (donc non rapportées), elles sont cependant accompagnéees d'une modalisation renvoyant au dire d'autrui («modalisation en discours second » : selon - d'après, aux dires de - Paul, Marie passera ses vacances en Italie) ».

20 L'article « Discours rapporté », signé de D. Maingueneau, in Charaudeau et Maingueneau (dir.) (2002) - renvoyant aux oppositions proposées in Authier-Revuz (1993a) - constitue une exception.

21 Cf. aussi Wagner et Pinchon (1991 : 308 sq.). ; Riegel et al. (1994 : 597 sq.) ; dans Détrie et al. (2001), l'article « Discours rapporté », ouvert par la définition très générale « Référence qu'un discours fait à un autre discours », se limite aux formes classiques du DA-objet, en mentionnant cependant (p. 94), de façon elliptique, les combinaisons de modes « grâce aux nombreuses formes de modalisation en discours second sur le contenu ou sur l'expression », le circuit des renvois amenant à « croiser » à nouveau, à l'article « Modalisateur » « ceux signalant l'utilisation d'un point de vue autre (pour/selon/d'après untel/ censément/soit-disant ; ...) » (p. 188). 
façon adéquate, à l'ensemble des « modalités » de cette intégration, comme objet ou comme source du discours « premier»;

- les présentations universitaires centrées sur l'énonciation - textualité, narration - enfin, qui, si elles mentionnent, à côté des formes « classiques » de DR - diversement articulées à celles-ci et sous des appellations variées : connotation autonymique, mise entre guillemets, îlots textuels, ... - des formes relevant bien, au plan des manières de dire, comme modalisation autonymique d'emprunt (MAE), de la modalisation par discours autre (MDA), font l'impasse sur le secteur, si riche et si présent dans les discours, de la MAS ${ }^{22}$;

La reconnaissance " aux côtés » du DR classique de formes relevant de la MAS est certes présente - depuis Bally (1914) - à travers de multiples occurrences, mais il apparaît que c'est - hors de toute visée d'inventaire historique exhaustif - de façon dominante, sur un mode intégrateur que se trouve pratiqué le rapprochement des formes de MAS au « discours rapporté », comme celui d'une « marge » à annexer au corps central de celui-ci ; telle l'assimilation, pratiquée de fait, par Kerbrat-Orecchioni (1980 : 162), par exemple, de X déclare, X estime que, selon $X$, etc. sous le nom, non-différenciant, d' "opérateur de discours rapporté », ou l'élargissement explicite du discours rapporté au-delà des frontières usuellement reconnues :

[...] les phénomènes de discours rapporté ne se limitent pas à ces trois stratégies [DD, DI, DIL]. [...] certains emplois du conditionnel [...] relèvent, indéniablement, du discours rapporté (Maingueneau 1981 : 98)

L. Rosier (1999 : 131)) enregistre ce mouvement, en évoquant « l'annexion des formes en selon $A$ et alii » et le fait que « le terme DR va progressivement englober une série de formes qui obligeront à le redéfinir lato sensu. ${ }^{23}$

22 Voir Herschberg-Pierrot (1993 : chap. 6-7), Perret (1994 : 97 sq.), Sarfati (1997 : 69), Jeandillou (1997 : 78), Stolz (1999 : 76 sq.), Tisset (2000 : 87-97), qui présentent, incidemment, une allusion à des formes de MDA, ne débouchant pas sur l'identification d'un type de RDA.

23 Ce que souligne G. Schrepfer-André (2004 : 576) depuis la perspective opposée, différenciatrice, qui est la sienne («Information référencée [par selon] versus discours rapporté ») : «Les selon $X$, d'après $X$ et pour $X$ de ce type sont le plus souvent considérés, aux côtés d'autres formes apparentées (suivant $X$, aux dires de $X$, si l'on en croit $X$, aux yeux de $X$, etc.) comme des introducteurs de discours rapporté », en étayant son constat d'une série de références allant de Bally (1914) à Rosier (1999). Notons qu'il serait utile, dans cet ensemble de références, de démêler, dans les rapprochements effectués des deux versants de la RDA, ce qui relève de l'amalgame « indifférenciant ", ou bien (ce qui n'est pas du même ordre, même si la différence entre les deux types de formes n'y est pas explicitée) d'un regroupement, fait explicitement, sous un angle spécifique, au 
La prise en compte adéquate de ces formes de MAS, dans leur rapport avec le « DR » au sens classique du terme, relève de leur rapprochement différenciateur. Ce double mouvement - marquer qu'elles appartiennent à un même champ, celui des formes renvoyant, dans le dire, à un discours autre et qu'elles s'opposent, par le statut qu'elles donnent à ce discours autre dans le dire, celle de l'objet dont il parle ou de la source d'après laquelle il parle - s'accommode très mal du poids terminologique de l'appellation « discours rapporté " $^{24}$. À partir du moment où les descriptions de ce champ reconnaissent pleinement ${ }^{25}$ - et non plus marginalement - l'ensemble des formes de la MAS $^{26}$ comme partie prenante, il paraît préférable de renoncer au terme unique « discours rapporté », condamné à osciller entre son sens classique, restreint au discours autre pris comme objet (DD, DI, DIL) et un sens qui n'est pas seulement « élargi » par rapport au premier, mais en rapport d'hyperonymie avec celui-ci : incluant, au plan de son extension, les faits de renvoi à un discours autre source et se réduisant, au plan de sa compréhension, à la représentation d'un discours autre.

Aux incertitudes - ou acrobaties - terminologiques liées à un terme unique renvoyant à des phénomènes de niveau différent ${ }^{27}$, la spécification systématique « DR au sens strict/au sens large ») apporte, certes, une désambiguïsation, mais, outre son incommode lourdeur, présente l'inconvénient d'affecter le versant « modalisant » de la RDA d'une sorte de caractère secondaire ou marginal, reflétant le caractère récent et encore partiel de sa prise en compte plutôt qu'une propriété linguistique.

Dans la mesure où ce qui reçoit un nom se trouve par là même doté d'une réalité plus « consistante », le souci terminologique ne peut être mineur... : c'est à faire apparaître, dans les appellations elles-mêmes, la partition d'un champ

nom d'une propriété commune, comme, par exemple, celui, proposé par J.-M. Adam (1990) des « marques d'attribution de proposition » dans lesquelles figurent « formes du discours relaté » [DD, DI, DIL] et formes selon $l$, pour l, à son avis.

24 Indépendamment des critiques déjà formulées sur le « rapport » consistant à imaginer, prévoir, etc. des propos jamais formulés.

25 Comme c'est, par exemple, le cas dans les parcours d'ensemble de Authier-Revuz (1992a, 2004a), Rosier (1999), Van Raemdonck (2002), Marnette (2005).

26 Et, au-delà, de la MDA, incluant, à côté des assertions (MAS), les manières de dire (MAE) empruntées $-c f$. ci-dessous.

27 Dans Haillet (1998), par exemple, on passe d'un sens à l'autre de « discours rapporté » : la mise en regard du « conditionnel d'altérité énonciative [avec] les formes du discours rapporté » entendu au sens (restreint) de « ce qu'il est convenu d'appeler le discours rapporté (et notamment avec ses trois sous-catégories [DD, DI, DIL])» (p. 63, 72) débouchant sur la conclusion que ce conditionnel est « par conséquent une forme de discours rapporté », au sens large. (p. 78, gdt). 
global de RDA selon une opposition entre DA-objet (le DR au sens « classique ») et DA-source (modalisant) que répond la terminologie proposée ici.

(iii) Autres approches de la partition DA-objet/source du dire.

Appréhendable aux plans syntaxique, énonciatif, sémantique, la partition ainsi tracée dans le champ de la RDA relève d'une différence structurelle, en langue, dont il importe à mon sens de ne pas perdre le caractère général et le « tranchant », dans des généralisations - arbitraires - de valeurs particulières (Bally), ou dans des approches décalées vers un plan qui n'est plus celui de la langue en tant que système de formes (Ducrot, Perrin).

- Bally (1914) C'est dans une perspective explicitement différentielle que se place Bally (1914) : " pour comprendre mieux » ce qui caractérise les « trois types grammaticaux », « servant à reproduire les paroles ou les pensées d'un tiers » («styles » direct, indirect et indirect libre), il convoque, au titre de « type grammatical » autre, " où il y a également reproduction d'un énoncé par [L] », des formes de MAS (D’après les dépêches du soir... ; À l'entendre... ; On aurait retrouvé les traces...). À cette différence, posée d'abord au plan grammatical, il fait correspondre, au plan sémantique, une opposition entre la « reproduction objective de E », qui caractérise le premier groupe, et une dimension « subjective » inhérente au second.

Peut-être n'est-il pas aberrant de rapprocher de cette formulation l'opposition - telle qu'elle a été envisagée ci-dessus - entre les deux positions qu'occupe, dans un Dire, le discours autre qui y est représenté : objet de la parole, dont elle parle, d'une part, et source de celle-ci, d'après/avec laquelle elle parle, d'autre part, " altérant » le mode subjectif « simple » de la prise en charge « ordinaire » (sans MAS) de ses assertions?

Mais il est clair que cette opposition structurelle entre reproduction objective (DA comme objet) et subjective (DA comme source modalisatrice) - si tant est qu'on admette, comme je l'envisage hypothétiquement ci-dessus, d'en déceler la présence dans l'analyse de Bally - est recouverte par une succession - glissade ? - de formulations stylistico-psychologisantes, aboutissant, pour la MAS, à privilégier - indûment - « une attitude personnelle [subjective] critique, sceptique même » de la part de $\mathbf{L}^{28}$, et, plus encore, de passer pour les DD-DI-DIL d'une caractérisation posée, par contraste, comme « reproduction objective de

28 Là où, hors de contextes spécificateurs (D'après $X$, toujours aussi mythomane, $P$ vs Selon des sources fiables, $P$ ), la suspension de prise en charge, de la part de $\mathbf{L}$, par la MAS - neutre par elle- 
E » (Bally 1914 : 410) à son inacceptable transformation en une sorte d'objectivité psychologique d'un énonciateur $\mathbf{L}$ « neutre », aussi dénué d'appréciation sur ce qu'il rapporte qu'un « phonographe» (ibid. : 422) :

pas moyen de croire que $\mathrm{R}[=\mathbf{L}]$ mêle quelque chose de ses pensées à celles de $\mathrm{S}[=\boldsymbol{l}]$; surtout pas trace d'une appréciation personnelle de ces paroles, de ces pensées (ibid. : 421).

- Ducrot (1980) L’analyse sémantique proposée ci-dessus (1.1.1), opposant les énoncés $\boldsymbol{l}$ dit que $P$ et selon $\boldsymbol{l}, P$, comme, respectivement, " parlant d'un dire (qui parle du monde » et " parlant du monde (d'après un dire) », se situe à un plan formel, en deçà des visées argumentatives, rhétoriques... dont, en discours, elles peuvent être le vecteur, comme des interprétations qu'elles peuvent recevoir.

Aussi, lorsque Ducrot (1980 : 44-46) pose, pour un énoncé de la forme $\boldsymbol{l}$ dit que $P$, l'alternative d'un fonctionnement relevant « du discours rapporté »ou de la polyphonie « selon le but attribué à $\mathbf{L}$ quand on interprète son énoncé », se situet-il à un tout autre plan : selon, en (23), que l'énoncé (a) « est dit pour favoriser une conclusion », ou « un enchaînement » de type b1 ou b2 :

(23) a Pierre m’a annoncé que le temps s’améliorerait.

b1 Pierre est un optimiste invétéré.

b2 J'irai à la campagne demain.

il sera analysé (a-b1) comme un " discours rapporté » dont « le propos » est les paroles de Pierre (selon une analyse proche de celle proposée pour $l$ dit que $P$ ci-dessus), contrairement à (a-b2), « polyphonique, dont le propos est l'amélioration $[\mathrm{du}]$ temps et non pas les paroles de Pierre » (ce qui rapproche ce cas de l'analyse proposée pour selon $\mathbf{l}, P)^{29}$.

Relativement à une forme de la structure $\boldsymbol{l}$ dit (que) $P$, l'analyse formulée par Ducrot :

même -, ouvre interprétativement sur tout l'éventail des valeurs épistémiques, en fonction des contextes et des co-énonciateurs.

29 Évoqué à l'orée d'une discussion critique par De Mattia (2000 : 107 sq.), le point de vue logique de Port-Royal sur « l'ambiguïté » de structures $l$ dit que $P$ « qui peuvent être prises différemment selon le dessein de celui qui les prononce » est très voisin de l'approche par Ducrot du «but » attribué à l'énonciateur. Ainsi, pour l'énoncé Tous les philosophes nous assurent que les choses pesantes tombent d'elles-mêmes vers le bas, la proposition principale - au sens grammatical du terme, l dit que - ne sera analysée comme « principale » que si le « dessein » est de rapporter l'opinion des philosophes ; sinon, elle « ne sera qu'incidente » à l'affirmation touchant aux choses pesantes. (Arnaud, Nicole (1660: 129)) 
On a discours rapporté si le but attribué à $L$, quand on interprète son énoncé, est de faire savoir ce qu'a dit L' [notre l] (p. 44)

est radicalement étrangère au plan où se situe l'opposition formelle entre nos deux versants de $\mathrm{RDA}^{30}$ : quels que soient les avatars interprétatifs d'un DI ayant pour objet un dire autre, et quel que soit l'intérêt - certain - de faire apparaître les " chemins interprétatifs » permettant à $\mathbf{L}$ d'avancer « $\mathrm{P}$ » sous couvert de parler du dire de $\boldsymbol{l}$, notamment dans le registre de « l'argument d'autorité » « on ne quitte pas le champ du DI pour autant $»^{31}$.

Notons d'ailleurs qu'il en irait de même, à mes yeux, pour la structure $P$, selon l:

(24) D’après Pierre, le temps va s’améliorer.

qui, autant que (23), est propre à recevoir, en discours, les enchaînements (b1) et (b2), celui-ci centré sur l'assertion P (l'amélioration du temps), celui-là se focalisant sur le dire de $\boldsymbol{l}$ convoqué en (24) à titre de source de l'assertion. Face à l'interprétation (24-b1) - mettant au premier plan ce qui, formellement, en (24) a le statut d'élément - second - modalisateur de l'assertion, « on ne quitterait pas » non plus, selon moi, le champ de la MAS.

- Perrin (2000,2004) Situées explicitement dans le sillage des analyses de Ducrot, les propositions de Perrin relèvent radicalement du niveau d'une réalité interprétative - le sens attribuable à un énoncé en contexte - appréhendée au-delà de ce qui, de façon insistante, est caractérisé comme « l'apparence » des formes. Ainsi, au nom du fonctionnement à titre « d'argument d'autorité » (l dit que $P$ comme façon - autorisée - de dire P), les formes relevant $\mathrm{du}$ " discours rapporté » se trouvent-elles scindées en « discours rapporté référentiel » (ou « simple discours rapporté ») et « discours rapporté modal » (appelé aussi «pseudo-discours rapporté » $2000: 90)^{32}$; cependant que, dans la même logique, se trouve posée une «forme de modalité » dite «allusive », sous laquelle se trouvent regroupées des formes comme il paraît

30 Cf. cette remarque de Recanati $(1981: 220):$ « [...] pour Ducrot, la notion de discours rapporté n'est pas comme pour nous une notion formelle : le même énoncé "Pierre dit que Henry est généreux" peut être interprété, selon lui, en termes de "polyphonie" ou en termes de "discours rapporté" suivant la façon dont le locuteur poursuit son discours (in Ducrot et al., 1980 : 44-46) ». 31 Comme conclut pertinemment De Mattia (2000 : 138) sur cette question.

$32 C f$. "Quelle que soit la forme apparente de la phrase en surface, même lorsqu'un verbe de parole introduit une phrase complétive à valeur de séquence rapportée, ce verbe peut avoir une valeur parenthétique à mon sens et la séquence rapportée se voir attribuer une portée modale. » (2004: 70) 
que, comme ma fille dit, le conditionnel... (relevant pour nous, formellement, de la MDA) et des formes qui, par delà leur « apparence » de DD, DI, sont assimilées aux premières ${ }^{33}$.

Parcourant les mêmes champs de données, les oppositions et catégories que nous venons d'évoquer (Ducrot-Perrin) et celles qui ont été proposées ci-dessus ne relèvent pas du même plan d'analyse du langage : l'opposition entre discours autre objet vs source posée ci-dessus comme structurant le champ de la RDA - opposition discrète entre deux structures formelles définies différentiellement - relève du plan de la langue comme système fini de différences ; les différenciations entre «DR référentiel et modal » ou la mise au jour d'une " modalité allusive ", qui se situent au plan du sens des occurrences concrètes en discours - sens singulier, inépuisable... - relèvent de balisages posés sur le continuum des interprétations en discours ${ }^{34}$.

La légitimité des deux plans d'analyse est évidente, chacun correspondant à un niveau du « réel » langagier ; mais il importe de ne pas les confondre, et cela d'autant plus que la question langagière «cruciale » est bien celle des « chemins » selon lesquels ces deux plans se « nouent », c'est-à-dire selon lesquels la langue - en discours - signifie.

De ce point de vue, je n'adhère pas aux formules qui, conférant au réel de catégories de langue un statut d' « apparence » (apparence de DR, apparence de « rection » derrière laquelle joue, en fait, un « parenthétique »), tendent à « fantômiser » le fait de la langue et de ses différences.

33 Ainsi dans cet exemple attesté des propos d'une mère de famille (2000: 90) : « Les enfants riaient bien je voulais prendre ma liberté. [...] C'est pas que je les aime pas mes enfants mais tusais tu aimes à vivre toute seule. Fait-que [alors] là les enfants riaient. Ils ont dit : "Maman veut prendre sa liberté" [...] » (soulignements et italiques dans le texte), le dernier énoncé est analysé comme « une séquence explicitement rapportée, “Ils ont dit : 'Maman veut [...]'”, mais où la mère ne fait allusion à ce qu'on dit ses enfants que pour qualifier implicitement son propre discours. Formellement cet énoncé a toutes les apparences d'un simple discours rapporté au style direct. Mais il ne faut pas s'y tromper. Loin de se contenter de rapporter ce qu'ils ont dit à son sujet, la mère s'exprime ici par la voix de ses enfants, sous leur autorité polyphonique. Une telle interprétation pourrait être explicitée par une incise du type "comme disent les enfants" [...] ».

34 On relira les précieuses pages de L’Amour de la langue (Milner (1978: 64-65)) distinguant la linguistique par son objet, la langue, « réel » « marqué du discernable », des « diverses disciplines herméneutiques » introduisant « des coupures », des « réseaux de discernement » « sur un objet qui les ignore », un « flux » [...]. 


\subsection{Mise en rapport du Dire avec un discours autre « conjoint » au Dire}

Au-delà de l'ensemble des formes de MAS - suspendant la prise en charge d'une assertion par son renvoi à un discours autre " source » - s'ouvre un vaste espace de formes énonçant le Dire en rapport avec un discours autre : champs voisins qu'il importe de ne pas confondre. On comparera d'abord (1.2.1) le fonctionnement des deux formes selon $\boldsymbol{l}, P$ et comme le dit $\boldsymbol{l}, P^{35}$, pour, derrière leur apparente proximité sémantique, faire apparaître la différence du mode sur lequel le discours autre intervient dans le Dire : mise en jeu, modalisante au sens strict d'un discours autre comme source de l'assertion (MAS) pour l'un, là où, pour le second, cela relève d'un accompagnement « circonstanciel » du Dire par un discours autre " conjoint », avant d'évoquer et d'interroger sommairement (1.2.2) la variété des formes par lesquelles le Dire se met « en rapport » avec un autre discours.

\subsubsection{Selon $\boldsymbol{l}, \boldsymbol{P}$ vs comme le dit $\boldsymbol{l}, \boldsymbol{P}$}

$\mathrm{Si}$, dans certains contextes de révérence incontestée à un certain $\boldsymbol{l}$ - le président Mao, les Saintes Écritures, Lacan,... les deux constructions :

(25) a) Selon $\boldsymbol{l}, \mathrm{P}$

b) Comme le dit $l, \mathrm{P}$

peuvent, interprétativement, au titre de l'argumentation par autorité, être reçues comme deux variantes du dire " garanti » par $\boldsymbol{l}$, de $\mathrm{P}^{36}$, la comparaison de leur fonctionnement interdit l'assimilation de cette subordonnée méta-énonciative ${ }^{37}$ de comparaison comme le dit $\boldsymbol{l}, P$ à la modalisation en assertion seconde.

On note, tout d'abord, par opposition aux faits de figement ${ }^{38}$ en dire, observables dans certaines formes de MAS, telles

35 C'est seulement en rapport avec selon $\boldsymbol{l}, P$ que comme le dit $\boldsymbol{l}$ est envisagé ici : la description, tant linguistique que discursive qu'appellent les divers emplois de cette circonstancielle comparative de RDA ne relève pas du projet de cet ouvrage - notamment la complexité de son fonctionnement « biface » vers l'amont et vers l'aval du dire, du type : $P$, comme le souligne $l:$ : $Q$ » : Cette décision a été une erreur, comme l'explique $\boldsymbol{l}:$ : Il ne fallait pas [...] ».

36 On a vu ci-dessus (1.1.2 p. 84) comment, en se situant au plan de l'interprétation en discours, c'est déjà la différence structurelle entre $\boldsymbol{l}$ dit que $P$ et selon $\boldsymbol{l}, P$, qui pouvait être escamotée.

37 Comme pour selon ( $c f$. note 6 ci-dessus) la subordonnée comme le dit $l$ ne relève pas univoquement du plan méta-énonciatif de l'incidence au dire de $\mathrm{P}$ (à un je dis implicite, si l'on veut) mais peut - générant des cas d'ambiguïté - s'articuler en circonstancielle, notamment de manière, au contenu de P : « Il repeint tout, comme le dit Jean. [(a) à la façon dont le dit Jean / b) Jean le dit aussi].

38 Dont le caractère inégal ( $c f$. ci-dessus, Remarque 2) serait à préciser. 
à ce que dit $\mathrm{l} P \mathrm{vs}$ * à ce que répète $\mathrm{l}, P$ aux dires de $l, P$ vs ${ }^{\star}$ ? aux confidences de $l, P$

que la catégorisation du dire autre convoqué au titre de comparant du Dire, déploie la plus grande richesse lexicale pour le verbe de parole

comme le dit, l'écrit, l'affirme, le démontre, le note, le signale, le souligne, le rappelle,... le concède,... le hurle, le murmure...

ou (avec des P niés, questionnés...)

comme le prétend, le soutient, l'imagine, l'assène, le radote...

Au-delà les deux types s'opposent, tant au plan de la nature du $\mathrm{P}$ " affecté » par un autre discours (i) qu’à celui de la prise en charge de $\mathrm{P}$ par $\mathbf{L}$ (ii).

\section{(i) Nature du P affecté par le discours autre : contrainte vs libre}

Lélément $\mathrm{P}$ mis en rapport avec un discours autre n'est soumis à aucune des contraintes évoquées ci-dessus quant au P modalisé en MAS :

- au plan temporel, modal, de la polarité, le dire qui « accompagne » le Dire peut - contrairement au DA source qui doit être réalisé (cf. (14), (15)) - être représenté comme futur, hypothétique, nié :

(26) Ça a été un progrès décisif, comme on le dira sûrement plus tard, comme il pourrait peutêtre le dire, comme j'aurais déjà dû le dire, comme il ne l'a jamais dit,... * selon ce qu'il n’a pas dit.

- au plan illocutoire, le Dire accompagné d'un autre dire peut - contrairement à la restriction assertive du selon $\boldsymbol{l}, P(c f .11,12)$ - être librement injonctif (27), interrogatif ${ }^{39}(28)$, exclamatif (29) :

(27) Viens donc avec nous comme te le dit/conseille Jean $v s$ *Selon Jean, viens donc avec nous.

(28) Comme le dit/demande Jean, qui croire dans cette affaire ? $v s{ }^{\star}$ Selon Jean, qui croire dans cette affaire?

(29) Comme le dit Jean, bravo, quel triomphe ! vs *Selon Jean, bravo, quel triomphe!

\section{(ii) Prise en charge de P par L : altérée vs stable}

Au plan de la prise en charge de $P$ par l'énonciateur $\mathbf{L}$, dans les environnements respectifs de MAS (selon $\boldsymbol{l}, \ldots$ ) et de mise en rapport avec un autre dire (comme le dit $\boldsymbol{l}, \ldots)$, l'opposition est radicale.

$39 C f$. (12) sur la valeur de selon + interrogation. 
En $P$, selon $\boldsymbol{l}$ et, de façon générale dans l'ensemble des formes qui relèvent de la stricte modalisation de l'assertion comme seconde (MAS), $\mathbf{L}$ se démet de la prise en charge de l'assertion de $\mathrm{P}$, remise à la responsabilité de sa source $\boldsymbol{l}$. Ce que $\mathbf{L}$ prend en charge c'est cette attribution de $\mathrm{P}$ à $\boldsymbol{l}$, et non l'assertion P elle-même, qu'il peut, librement, partager, mettre en doute, contredire :

(30) Marie a été imprudente, selon Jean ;

a) je trouve qu'il a raison.

b) personnellement, je n'ai pas d'avis.

c) ce n'est pas juste, elle a joué de malchance.

En $P$, comme le dit $\boldsymbol{l}$, en revanche, $\mathbf{L}$, qui ne se démet pas de la prise en charge de $\mathrm{P}$, l'assume pleinement comme son dire et, d'autre part, le met en rapport (ici de comparaison...) avec un autre dire ; aussi les enchaînements a), b), c), naturels en (30), relèvent-ils, en (31), de l'étrangeté tautologique ou du renforcement emphatique pour a), de l'incohérence pour b) et de la contradiction du type $P$ et non $P$ pour c) :

(31) Marie a été imprudente, comme l'a dit Jean ;

a) ? et il a raison.

b) * personnellement, je n'ai pas d'avis.

c) * ce n'est pas juste, elle a joué de malchance.

L'opposition quant à la prise en charge, ou non, de l'élément $\mathrm{P}$, par L, dans ces deux constructions se manifeste dans le statut que des je dis $P$ y reçoivent. Dans $P$, comme le dit $\mathbf{l}$, P étant normalement pris en charge par $\mathbf{L}$, il est possible de le « préfixer » d'un je dis de redoublement méta-énonciatif (ARD) :

(32) Je dis P, comme le dit $\boldsymbol{l}$.

Écoute, moi je te dis, comme il te l'a dit plusieurs fois : ra-len-tis un peu tes activités. (Conv. 17-02-2015)

L'énoncé présente alors la succession ARD-RDA d'un Dire d'abord emphatisé par son auto-représentation, puis mis en rapport par comme avec une RDA partageant le même $\mathrm{P}^{40}$.

Rien de tel avec

40 Succession à comparer avec celle de deux prédications de discours autre, reliées par la modalisation (en comme) de la première (principale) par la seconde (subordonnée) partageant le même P : ${ }_{1}$ [Il a dit que ${ }_{\mathrm{p}}$ (c'était beau)] comme ${ }_{2}$ [tout le monde (le) disait], comme en : "J'aurai à mes gages toute la troupe villemorienne et je leur dirai, comme on me l'a dit, « Allons faquins, que l'on m'amuse » [...]" [Diderot, Le Neveu de Rameau]. 
(33) Selon $\boldsymbol{l}$, je dis $\mathrm{P}$.

Selon lui, je dis qu'il faut renoncer.

où le je dis, dépourvu de toute dimension méta-énonciative par rapport au Dire en train de se faire énoncé par $\mathrm{L}_{\mathrm{i}}$, relève de la reformulation par $\mathrm{L}_{\mathrm{i}}$ d'un énoncé antérieur (source) de $\mathrm{l}_{\mathrm{i}}$, consistant en une forme de RDA du type :

(34) $\boldsymbol{l}_{i}$ dit qu'il faut renoncer.

à l'origine du « je dis » relevant aussi de la RDA, et non de l'ARD de (33).

La différence au plan de la prise en charge de P par $\boldsymbol{l}$ entre ces deux formes se manifeste aussi lorsque, via les pauses et décrochements, intonatifs ou typographiques - entre parenthèses ou double tirets, après le point final - ils apparaissent, dans le cours du dire comme mouvement second : là où celui-ci revêt pour le premier (MAS) une valeur, volontiers ostentatoire, de rectification touchant la valeur de vérité de l'assertion $P$ pour $\mathbf{L}$, il relève ( mise en rapport avec un autre discours) de l'ajout d'une information par rapport à celle - non mise en cause - apportée par P.

(35) a) L'usine va s'installer par ici, selon Jean.

b) L'usine va s'installer par ici, comme Jean le dit.

Ainsi détaché, le mouvement second de prise de distance d'avec l'assertion P de la MAS peut-il être souligné, par enfin, $d u$ moins... contrairement à ce qu'il en est avec l'accompagnement par la représentation d'un autre dire :

(36) a) L'usine va s'installer par ici, enfin selon Jean, du moins selon Jean.

* L'usine va s'installer par ici, enfin comme Jean le dit, du moins comme Jean le dit.

b)

le caractère d'après-coup correctif de la MAS pouvant se trouver explicité, comme en :

(37) - Ces peines-là ne guérissent pas si vite, répondit la petite Fadette ; et puis, se ravisant : Du moins à ce qu'on dit, fit-elle [G. Sand, La petite Fadette, chap. XX].

(38) - La signora Goosens avait une sœur qui a vécu un certain temps ici et qui, au dire de tout le monde, était un personnage désagréable, jaloux et malveillant. Du moins, se corrigea-t-il, selon la Signora Giusti [M. Nabb, Mort d'un orfèvre, traduit de l'anglais, 10/18, 2001] (ici, avec une récursivité de la MAS).

Remarque 3 : MAS vs mise en rapport avec un autre dire dans l'espace détaché de la note. L'opposition entre $P$, selon $\boldsymbol{l}$ et $P$, comme le dit $\boldsymbol{l}$ au plan de la prise en charge de $\mathrm{P}$ par L, altérée pour le premier et non pour le second, se traduit au plan de leur distribution : si l'une comme l'autre figurent également dans les formes de « décrochement » typographique sur la ligne (parenthèses, double tiret) (cf. le à ce que dit l'histoire de La Fontaine, note 9, par exemple), il n'en va 
pas de même pour leur apparition « en greffe » de la ligne du dire, dans cet autre espace paginal qu'est la note ${ }^{41}$; là où l'ajout d'un autre dire en rapport avec le dire de $\mathrm{P}$ y figure sans restriction (cf. par exemple dans ce texte même, les notes 94-95 du chap. 8 : comme le souligne $\mathrm{X}$, comme le note $\mathrm{X}$ ), la modalisation en selon, d'après, y est restreinte aux cas où la note va dans le même sens que $P$, notamment dans l'indication en note de la référence précise au discours sur lequel s'appuie le dire de P, comme dans cet exemple emprunté à l'étude de J. Lefèbvre (2007 : 119) :

Un enfant de six ans sur cinq souffre d'un trouble de la vision, à son entrée à l'école primaire ${ }^{(1)}$.

- . - -

(1) Selon une enquête réalisée en 1999-2000 sur plus de 30000 élèves à partir des examens de santé à l'entrée en primaire, par la Caisse primaire d'assurance maladie de Paris [Valeurs mutualistes 221, p. 26/27].

\subsubsection{Diversité des mises en rapport Dire/discours autre conjoint}

La comparaison, ci-dessus, des fonctionnements de selon $\boldsymbol{l}$ et comme le dit $\boldsymbol{l}$, pris comme représentants respectifs de chacun des deux types de construction - MAS et mise en rapport Dire/discours autre conjoint - par lesquels le Dire est affecté, au plan du contenu, par un discours autre a permis ${ }^{42}$ de dégager les points essentiels sur lesquels s'opposent ces deux ensembles : la nature du P auquel la modalisation est incidente et l'impact de cette modalisation sur la prise en charge de $P$ par $\mathbf{L}$.

Ainsi la liberté - temporelle, modale, illocutoire... - de P, comme la stabilité de sa prise en charge par $\mathrm{L}$, dans le cadre de sa modalisation par mise en rapport avec un autre discours, sont-elles partagées par toutes les formes de cet ensemble.

En revanche, si cette forme comme le dit $\boldsymbol{l}$ - avec le verbe dire ou tout autre verbe de parole - est, de très loin la plus fréquente, en discours, elle n'est représentative de l'ensemble hétérogène des formes de mise en rapport du Dire avec un discours autre ${ }^{43}$, ni au plan de la diversité formelle à travers laquelle se réalise cette mise en rapport, ni à celui de la variété des rapports représentés entre les deux discours « conjoints ».

41 Sur ces deux dispositions typographiques de la parenthèse-double tiret et de la note infrapaginale, je renvoie aux corpus et analyses respectivement de S. Pétillon-Boucheron (2002) et de J. Lefèbvre (2007).

42 Facilitée, on l'a dit, par l'apparente proximité sémantique entre le Dire selon et le Dire comme, que ne présente pas, par exemple, pour aller contre ce que $\mathbf{l}$ vient de dire...

43 Identifiable, dans le champ immense des éléments circonstanciels méta-énonciatifs, incidents non au dit $\mathrm{P}$ mais au fait de dire $\mathrm{P}$ - pour ne rien te cacher, soit dit en passant... etc., jusqu'au si tu as soif, il y a de la bière au frigidaire, analysé par Ducrot (1972 : 176), comme sous-ensemble référant à un discours autre. 
Se distinguent ainsi en terme d'information donnée sur le discours autre, les trois types :

(a) $P$, comme le dit $\mathbf{l}$ où l'anaphorisation de $\mathrm{P}$ par le, interne à la phrase, spécifie maximalement le discours autre, comme de contenu semblable à $\mathrm{P}$.

(b) P relateur ce que dit $\mathbf{l}$, où le ce que (contrairement à le) ne dit rien du contenu du dire autre, renvoyé, à l'extérieur de la phrase, à une interprétation plus ou moins aisée ( $c f$. ce que vient de dire vs ce qu'on a pu dire), l'information sur le dire autre se réduisant à l'indication de sa relation avec $\mathrm{P}^{44}$ comme

- contraire au contenu de $\mathrm{P}$ :

contrairement à, en désaccord avec, pour aller contre, à rebours de... ce que dit $\boldsymbol{l}$;

- conforme ou compatible, souvent inscrit dans un enchaînement avec P : conformément à, d'accord avec, à la suite de, dans le sillage de, en écho à, pour suivre, pour rebondir, renchérir sur... ce que dit $\mathbf{l}$ :

- ou, sans indication de contenu, par sa seule position dans une interaction : en réponse, pour répondre... à ce que dit $\mathbf{l}$

(c) P relateur $\mathbf{l}$, où c'est le fait même d'un discours autre qui n'est pas explicité : l'interprétation méta-énonciative modalisante de la construction demandant alors (majoritairement ${ }^{45}$ ) que l'énoncé soit explicitement situé au plan du Dire : soit par la préfixation de $\mathrm{P}$ par je dis (ou autre verbe de parole) :

(39) Je dis donc

On dira

Je dirai

Observons pour conclure à la suite de $\quad \boldsymbol{l}, \mathrm{P}$

dans le sillage de

en accord avec

avec

soit par le caractère explicitement métalangagier de la mise en relation :

(40) En réponse à $\quad l, \mathrm{P}$

Pour répondre à

Aussi, beaucoup moins spécifiant que celui, dans comme le dit $\boldsymbol{l}$, de comparant (d'identité) pour le Dire de $\mathrm{P}$, le statut sémantique du discours autre " lié », « conjoint » au Dire dans l'ensemble du champ de ces mises en rapport du Dire avec un discours autre, apparaît-il seulement, comme celui d'un point de repère à partir duquel, en référence auquel, le Dire peut se situer, se positionner.

44 Préférentiellement précédé d'un je dis pour certains comme en désaccord avec, d'accord avec...

45 Hors de mon propos ici, l'étude distributionnelle fine d'un corpus plus large serait nécessaire pour préciser - et peut-être limiter - cette contrainte. 


\subsubsection{Discours autre source (MAS) vs discours autre conjoint}

Ainsi les deux ensembles de formes convoquant un discours autre dans le Dire, du type :

- selon, pour $\mathbf{l}, P$

et

- comme le dit $\mathbf{l} /$ à rebours de ce que dit $\mathbf{l}, P$

évoqués respectivement en 1.1 et 1.2.2 (a, b, c), s'ils « affectent » l'un et l'autre le Dire du contenu de $\mathrm{P}$, se distinguent, on l'a vu, aux divers plans

- de l'inventaire de leurs formes : clos vs ouvert,

- de leur fonctionnement : restreint vs libre, quant à la nature du $\mathrm{P}$ « affecté » par du discours autre,

- de leur incidence sur le Dire de P : modalisation par laquelle l'énonciateur se démet de sa prise en charge vs mise en rapport, de nature variée, du Dire de $P$ avec un autre discours,

- du statut sémantique conféré à l'autre discours : source, modale, de l'assertion $\mathrm{P} v s$ repère, second terme d'une relation entre $\mathrm{P}$ et un autre discours.

On peut reconnaître dans le premier un des cinq modes ( $c f$. chap. 9) par lequel la langue répond à la question de la « représentation du discours autre » : celui, sur le versant de la modalisation du Dire par du discours autre, de la MAS, « altérant » la prise en charge de l'assertion. Le second, en revanche, enrichit, étoffe le Dire de $P$ de la prise en compte de l'environnement discursif d'autres discours par rapport auxquels le Dire de $\mathrm{P}$ - non « altéré » si l'on veut, par cet accompagnement - se positionne.

Remarque 4 : Inscription phrastique de la MAS et du DA conjoint. L'articulation Dire/discours autre source de la MAS, peut certes, au-delà de sa réalisation de base, selon $\boldsymbol{l}, P$, se «distendre », mais dans des bornes fixées par un lien anaphorique entre phrases adjacentes ; la connexion Dire/discours autre conjoint semble, en revanche, pouvoir se déployer depuis les formes phrastiques de la subordonnée circonstancielle méta-énonciative du comme le dit $l, P$ jusqu'à des mises en rapport interphrastiques situées à un plan sémantico-discursif (étranger à la MAS).

Ainsi au fonctionnement circonstanciel régulier (complément ou subordonnée) du discours autre source de la MAS (a), comme à celui du discours autre conjoint (b), peut-on adjoindre, comme partie prenante de la construction phrastique :

- leurs occurrences détachées en après-coup par une pause intonative ou un point (avec ou non l'introduction d'un enfin, du moins... (cf. (35-38)),

- les phrases incidentes, notamment avec anaphore ${ }^{46}$, telles, s'apparentant respectivement (a) à d'après $\mathbf{l}$ :

$46 C f$. sans anaphore, la forme ...- $\mathbf{l}$ dixit - ... 
(41) La mairie va, je dis ça d'après ce qu'on m’a dit, remettre à plat le plan de circulation [oral, 15-05-2014].

(42) Le camping (je le tiens du gardien) va fermer l'an prochain [Corresp. 10-09-2000].

et (b) à comme le dit $\boldsymbol{l}$ :

(43) Rastignac, on l'a dit souvent, emprunte quelques uns de ses traits à M. Thiers [F. Marceau, Balzac, p. 47].

(44) Il vaut mieux, même si on dit le contraire, arroser au soleil que pas du tout [Conv., 14-082016)].

Par extension, il paraît raisonnable d'intégrer les phrases adjacentes reliées par une anaphore du P modalisé comme (a) MAS en mouvement second de rectification :

(45) Ce n'est même pas lui qui a choisi sa femme, alors rendez-vous compte. Enfin, c'est ce qu'on dit [F. Vargas, L'armée furieuse, 2011, p. 358].

(46) De loin, à voir ma dégaine svelte, on ne me donne pas mon âge. Du moins est-ce là ce qu'a affirmé Dido [H. Troyat, La Dérision, p. 30].

- $\quad$ aussi bien que comme (b) mise en rapport avec un DA :

(47) Formes diverses de la vie, toutes vous me parûtes belles. Ce que je te dis là, c'est ce que me disait Ménalque [Gide, Les Nourritures terrestres, éd. Pléiade, p. 158].

(48) L'immigration est une chance pour un pays, s'il sait l'accueillir. Évidemment, ce n'est pas ce que... disent majoritairement les médias [Oral, 01-05-2017].

En revanche, seules les mises en rapport (b) du Dire avec un discours autre conjoint peuvent se réaliser - explicitées par des connecteurs comme en revanche, au contraire, de même, de la même façon ; pareil... - dans des suites phrastiques, sans anaphore :

(49) Il faut renoncer. (D'ailleurs) $\boldsymbol{l}$ dit de même, qu'il ne faut pas s'entêter..

(50) Il faut renoncer. (Mais) $l$ prône, en revanche, la persévérance.

et non nécessairement adjacentes :

(51) Il faut renoncer. C’est une question de bon sens. $l$ dit de même/au contraire que...

Relativement aux énoncés (a)-(b)-(c) (cités en 1.1.1, exemples (4) et (5)) et caractérisés respectivement comme :

- parlant du monde :

Marie a fait une longue promenade.

- parlant d'un discours autre (DA objet-DI) :

Jean dit que Marie a fait une longue promenade. 
- parlant du monde d'après un discours autre (DA source-MAS),

(c) D’après Jean, Marie a fait une longue promenade.

le type $(d)$ :

(d) Marie a fait une longue promenade, comme le dit Jean contrairement à ce que dit Jean pour renchérir sur ce que dit Jean etc.

est constitué d'un élément (a) parlant du monde et d'un élément de type (b) parlant d'un discours autre, subordonné au premier.

Par rapport aux deux modes de RDA, distincts, du DI et de la MAS - figurant respectivement en (b) et (c), un énoncé du type (d) ne présente pas un autre mode de $\mathrm{RDA}^{47}$ mais l'intégration d'une RDA de type (b) dans une construction comme élément subordonné à valeur méta-énonciative, c'est-à-dire incident au Dire de la principale.

À ce titre, c'est dans l'étude du DI, comme faisant partie de ses variétés de réalisation et d'emploi, que ce fonctionnement - ce qu'on a appelé discours autre « conjoint » ou " mis en rapport » avec le Dire - mérite d'être décrit précisément.

\section{La modalisation d'une manière de dire par discours autre}

La configuration de la MDA n'est pas, on l'a vu au chapitre 1, cantonnée, au plan du contenu du dire ; elle concerne tout autant celui des formulations, des formes signifiantes, des « manières de dire » que $\mathbf{L}$ énonce en convoquant explicitement un discours autre : c'est ce qu'illustrent les couple de formes - (66) vs (67) ; (68) vs (69), (70-71) vs (72-73) du chap. 1 - renvoyant respectivement au contenu vs à la forme du Dire.

Comme à celui du contenu, on peut distinguer deux types d'incidence du discours autre au plan des manières de dire.

47 Cf. chap. 9.2, p. 338, la notion de mode de RDA, définie par une formule, abstraite, de traits distinctifs. 


\subsection{La modalisation autonymique d'emprunt : $X$, comme dit $I$}

De la même façon que, avec la MAS, le Dire, " passe » par l'assertion d'un discours autre, le Dire peut, en un point de son avancée, passer, pour nommer, par la manière de dire d'un discours autre, se démettant, par cet « emprunt » de sa propre manière de dire et, comme pour l'assertion en MAS, de la prise en charge qui s'y attache.

Ainsi, faut-il distinguer :

(52) Je voudrais bien lui /conter fleurette, comme disait grand-mère, et d'un peu près, à cette gonzesse. [conv., mai 1992]

où c'est la manière de dire désuète « conter fleurette » qui est énoncée par $\mathbf{L}$ comme empruntée à un $\boldsymbol{l}$ « source » (sa grand-mère) étranger à la situation, là où en :

(53) D’après ses copains, il aimerait la draguer.

c'est (MAS) l'assertion énoncée par $\mathbf{L}$, et non la manière de dire, qui est donnée comme reçue d'ailleurs.

De même que (53) doit être distingué de

(54) J'aimais bien quand grand-mère disait d'un air réjoui : Je crois qu'il lui conte fleurette !

où les mots de $\boldsymbol{l}$ (la grand-mère) sont, au DD, l'objet (et non la source) du dire de $\mathbf{L}$ (qui ne les « emprunte » pas pour en faire usage comme en (52)). Le fait que la grand-mère dise «conter fleurette » est, en (54), partie intégrante de l'objet du dire de $\mathbf{L}^{48}$, là où, en (52), il est convoqué au titre de source des mots - d'emprunt qu'il énonce.

On revient au chapitre $\mathbf{8}$ sur la très grande variété des formes par lesquelles se réalise la modalisation autonymique d'emprunt ${ }^{49}$, en notant seulement ici des éléments de comparaison avec le fonctionnement de la MAS.

Comme dans le cas de la MAS, cet élément apparaît - dans la mise en regard des énoncés (55) avec (52) - facultatif (a), mobile (b), multipliable (c) :

(55) a. Je voudrais bien lui conter fleurette, et d'un peu près [...].

b. Je voudrais bien, comme disait grand-mère lui /conter fleurette [...].

48 Dans le cadre de l'interprétation du « e » autonyme comme reproduction fidèle des mots de l, cf. chap. 8.2.2.2 p. 263.

49 Décrite systématiquement dans Authier-Revuz, (1995/2012 : 258-464, et Rappel-inventaire des formes : 259-260) ; notamment, outre $X$, comme dit $\boldsymbol{l}: X$, j'emprunte ce terme à $\mathbf{l}$, pour reprendre le mot de $\mathbf{l}$, selon les mots de $\mathbf{l}$, comme l'appelle $\mathbf{l}$, pour parler de manière vulgaire, pédante... ; ce que $\mathbf{l}$ appelle, baptise $X . .$. ; un $\mathbf{l}$ dit $X . .$. 
Je voudrais bien lui, grand-mère disait / conter fleurette [...]

c. Je voudrais bien lui /conter fleurette, comme disait grand-mère, et d'un peu près, /à cette gonzesse, pour parler comme toi.

En revanche, la MAE ne connaît pas les restrictions observées pour la MAS, quant à l'élément modalisé : comme dit $\boldsymbol{l}$, par exemple, peut modaliser, comme emprunté à un discours autre, n'importe quel type de " fragment de chaîne » - quelle que soit son statut grammatical et sémantique : du déterminant (56), par exemple, à la phrase entière, et sans restriction à un contenu assertif, (57) :

(56) «La » (comme on dit souvent) science n'existe pas, seules des sciences existent ${ }^{50}$.

(57) Qui m'aime me suive, comme a dit je ne sais plus qui !

Je m'ennuie... Qu'est-ce que je peux faire ? ... comme disait Anna Karina dans « Pierrot le fou .

On verra (chap. 8.3.3.2, p. 299) que la MAE se combine avec toutes les autres formes de RDA. Notons seulement ici le caractère très commun, du cumul d'une MAS et d'une MAE partielle (a) ou globale (b) portant sur la formulation de la première :

(58) a. D'après le maire, c'est le tout qu'il faut « détruire $»^{51}$

b. D’après le maire, « l'ensemble de la construction est à détruire ».

Notons que de la même façon que si la MAS nous est apparue comme relevant de l'intersection de la catégorie médiative/évidentielle (voire de la modalisation épistémique ou de la prise en charge) avec le champ de la RDA, la MAE apparaît comme la zone où la configuration méta-énonciative de la modalisation autonymique - celle d'un dire revenant réflexivement sur les mots mêmes dont il fait usage, dans leur singularité signifiante - rencontre la RDA : le discours autre y apparaît comme l'une des dimensions du fait langagier - à côté de l'équivoque, de l'écart entre les mots et les choses ou entre les interlocuteurs... - venant s'imposer en « modalité opacifiante » du dire, dans l'usage - ordinairement transparent - de mots énoncés comme « allant de soi $»^{52}$.

50 Sur la liberté « a-syntaxique » d'insertion de la modalité autonymique, $c f$. Authier-Revuz (1995/2012: $145 s q)$.

51 Le cas le plus courant de la coréférence de la source de la MAS (le maire) et de la MAE (portant sur « détruire »), qui pourrait être marquée par sic n'est pas une contrainte ; comparer :

(58) c. D’après le maire, il faut « tout foutre en l'air » comme tu dirais.

52 Cf. ci-dessous, chap. 8.3.2, p. 291. 


\subsection{Modalisation par couplage avec la manière de dire d'un discours autre}

Dans ce cas, il n'y a pas remplacement d'un élément du Dire par du discours autre, mais (comme précédemment au plan du contenu) mise en rapport, " couplage », ici, de deux manières de dire, celle du Dire et, la modalisant par leur mise en regard, celle d'un discours autre. Comparons les énoncés suivants : a) sans modalisation, b) avec $\mathrm{MAE}^{53}$ simple et c) avec modalisation par couplage :

(59) a) C'était vers la fin de la guerre. Un groupe de résistants était descendu au village.

b) C'était vers la fin de la guerre. Un groupe de « terroristes » comme il disait était descendu au village.

il disait « terroristes »

ce qu'il appelait « terroristes »

c) C'était vers la fin de la guerre. Un groupe de résistants - il disait terroristes - était descendu au village.

C'était vers la fin de la guerre. Un groupe de « terroristes » - c'est-à-dire de résistants était descendu au village.

Là où, en b) la MAE se substitue à une manière de dire de L, en c) la modalisation autonymique par couplage avec un autre dire consiste en une mise en résonance du Dire avec un autre discours se prêtant, à travers des formes diverses, à des rencontres aussi dissonantes qu'harmonieuses, à des effets de sens ${ }^{54}$ multiples, notamment selon que la mise en rapport du Dire et du discours autre se fait par un mouvement centripète, de l'autre vers soi, comme par exemple en (60), en effet « glossaire » (a) ou « parler vrai » (b) :

(60) (a) ... l'heure du souper arrive après none (après trois heures)... [ouvrage sur le Moyen-Âge] Il y aura un lien fixe (traduisez un tunnel) entre la France et l'Angleterre. [Infos télé., 1986]

(b) ... un poste libéré (licenciement). [texte préparatoire aux Assises de la linguistique, 1982] ... une « explication franche » comme on dit en langage diplomatique pour qualifier une forte engueulade.

ou par un mouvement centrifuge d'ajout - informatif, pittoresque, polémique, de fausse auto-correction... - de la manière de dire de l'autre à ce qui se trouve déjà nommé, comme en (61) :

53 Dans les trois constructions selon lesquelles elle peut se réaliser, $c f$. ci-dessous chap. 8, ex. (78) à (82), p. 290 et Authier-Revuz (1995/2012: 52-55).

54 Sur la diversité des formes et des mouvements - centripètes/centrifuges - des couplages de modalisation autonymique par discours autre, $c f$. Authier-Revuz (1995/2012 : 352-359) d'où proviennent les exemples ci-après, ou Authier-Revuz (2000b : 45-52). 
(61) a Au treizième siècle, les banquiers - on les appelait changeurs - étaient surtout des Italiens [...] [ouvrage sur le Moyen-Âge].

b Au pessimisme de $\mathrm{M}$. Jospin - on préfère parler de "réalisme » au parti socialiste répond naturellement l'optimisme de l'opposition [...] [Le Monde, 15.1.83].

c Les grandes idées patriotiques du ministre (eux disent « cocardières ») avaient beau être connues, le choc a été rude [La rentrée Chevènement (chez les instituteurs), Libération, 9-9-1985, p. 4].

d [un régime] où la torture des opposants politiques (pardon, des «traîtres ») et le châtiment collectif de leur famille ont force de loi [M. Kravetz, Libération, 10.9.90, p. 46].

Sans entrer dans le détail du fonctionnement de ces couplages de manières de dire de l'un et de l'autre - qui ne relève pas du propos de cet ouvrage - on peut noter que, relativement à la distinction opérée ci-dessus (1.2) entre stricte modalisation par un discours autre (MAS) et mise en rapport du Dire avec un discours autre, les deux trajets, centrifuge et centripète, ne sont pas équivalents : dans le premier, il y a bien une MAE, au sens strict, au même titre que dans les formes à nomination unique ( $c f .2 .1)$ puisque le Dire de $\mathbf{L}$, dans un premier temps, «passe » bien, en (60), par l'emprunt au discours autre de « libéré, none, lien fixe... », là où, dans le second, c'est seulement à titre d'ajout, on l'a dit, qu'une nomination, énoncée et prise en charge «normalement »-sans réflexivité opacifiante, comme « allant de soi »- se voit, secondairement, par sa mise en rapport « couplée », en (61), avec une manière de dire autre autonymisée (" changeurs, cocardière, traîtres »), affirmée dans sa spécificité signifiante de « manière de dire » propre.

En toute rigueur, le terme de modalisation autonymique d'emprunt, qui sera conservé pour l'ensemble des formes, n'est donc adéquat que pour deux des trois configurations évoquées :

1. la modalisation - de base - simple :

Voici un « X » comme il dit.

2. la modalisation couplée centripète :

Voici un « X » comme dit $l$ (c'est-à-dire un Y)

mais pas pour la dernière :

3. la modalisation couplée centrifuge :

Voici un Y (lui appelle ça « X »).

Remarque 5 : Sur l'extension abusive faite ici de MAE (d'emprunt) à toute MA avec DA. L'opposition entre :

A - modalisation stricte par discours autre source (MDA) affectant la prise en charge par L d'un élément de son Dire,

et 
B - mise en rapport (susceptible " d'effets modalisants ») du Dire avec un discours autre, a été posée comme jouant également au plan (1) du contenu et (2) de la forme du Dire. La distinction A/B n'est cependant pas, dans ce qui suit, prise en compte et marquée dans la terminologie au plan (2) de la forme comme elle l'est au plan (1) du contenu. Là où la MAS - modalisation en assertion seconde - a été nettement opposée à ce qui relève des « mises en rapport » avec un autre discours, renvoyées en dehors du versant MDA, la catégorie de la MAE, telle qu'elle est utilisée ici, renvoie ici, dans le cadre de la MDA, à tous les cas de modalisation autonymique impliquant du discours autre, y compris ceux qui, en couplage, ne passent pas directement par un emprunt : d'une part, l'abus de langage que cela constitue, répond à une certaine diffusion du terme MAE, et au caractère relativement marginal (par rapport à l'étendue des mises en rapport au plan du contenu) des couplages centrifuges ainsi « annexés »; d'autre part, inscrite dans le cadre étroit - « cellule » ou « bloc syntactico-référentiel - d'une nomination dédoublée (... X, $\boldsymbol{l}$ dit $Y$...), la « mise en rapport » Dire/discours autre semble impliquer une modalisation de la manière de dire propre au Dire, « altérée » par son couplage avec celle du discours autre, qui n'est pas le fait de l'ensemble des divers effets de rencontre, au plan du contenu, du Dire et de ses autres conjoints.

\section{Deux versants pour la RDA}

Le schéma suivant résume le partage opéré dans le champ de la RDA par le statut distinct conféré au discours autre représenté dans le dire, celui de DA-objet (prédication de discours autre) et celui de DA-source (modalisation par discours autre) :

[VII]

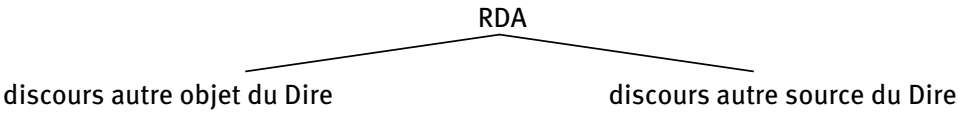

prédication du fait d'un discours autre

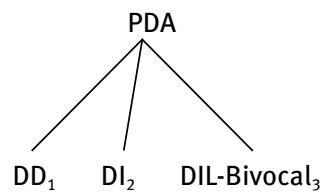

modalisation par un discours autre

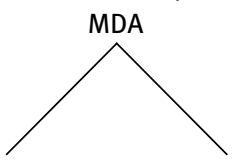

d'un contenu assertif d'une manière de dire

modalisation en assertion seconde modalisation autonymique d'emprunt

$\mathrm{MAS}_{4}$

$\mathrm{MAE}_{5}$

1. Il dit : je vais tous les battre !

2. Il dit qu'il va surclasser tout le monde.

3. Il ne doute de rien : Il va tous les battre !
4. À l'entendre, il va tous les surclasser.

5. J'espère qu'il va les « pulvériser » comme il aime à dire.

Au-delà des différences observables dont cette opposition formelle entre DA-objet $v s$ source du dire permet, directement, de rendre compte, c'est relativement à elle que l'on peut appréhender - et éclairer - des cas délicats que je ne fais ici que mentionner : 
- indétermination ou indécidabilité, comme, par exemple, pour une séquence « $\boldsymbol{e} »$ syntaxiquement autonome et sans marquage explicite, entre lecture en DD ou MAE, du type Le proverbe dit bien... ou Comme dit le proverbe...

(62) J'ai été trop gentille avec elle, c'est une punaise... [ton emphatique] Oignez vilain, il vous poindra... [conv., 5-5-2006]

- forme régulière de neutralisation, que réalise l'incise en dit-il, de l'opposition entre DD et MAE ( $c f$. (62)) dans :

(63) J'ai été trop gentille avec elle, c'est une punaise... Oignez vilain, dit le proverbe, il vous poindra.

aussi bien que celle entre DI et MAS de (64), en (65) :

(64) DI : On dit qu'il va se présenter vs MAS : A ce qu'on dit, il va se présenter.

(65) Il va, dit-on, se présenter.

On a indiqué comment ces deux versants de la RDA s'opposent aux plans sémantiques, énonciatifs, syntaxiques ; c'est aussi par rapport au fait langagier fondamental de l'hétérogénéité constitutive du dire ${ }^{55}$ ( $c f$. ci-dessous chap. 10) que prend sens l'opposition entre les RDA en parler de vs parler d'après un discours autre. Au regard du fait que, le dire se produisant dans le milieu du "déjà-dit » des autres discours, en est inévitablement traversé et issu, c'est, en effet, tout mot, tout énoncé - manière de dire, assertion - qui peut être " lesté » d'une origine discursive. Ainsi, si certains énoncés parlent d'un discours autre plutôt que de tout autre chose, c'est tout énoncé qui, foncièrement, parle d'après du discours autre : relativement à ce réel d'un dire décoïncidé de lui-même par l'extériorité discursive qui, de façon permanente, le traverse, le rapport à l'altérité discursive que marque la MDA - bémol " altérant », sur la portée du dire, telle assertion, telle manière de dire comme venue d'un ailleurs discursif - apparaît comme celui d'une émergence, d'une émanation de ce « terreau » du déjà-dit, plus proche que ne l'est l'opération constituant le discours autre en objet, distinct de son propre discours.

55 Dont l'un des axes - celui qui nous occupe ici avec la RDA - tient à cette extériorité interne que, différemment, le dialogisme bakhtinien et l'interdiscours de Pêcheux reconnaissent comme au principe même du dire ( $c f$. Authier-Revuz 1982a, 1984, ou 1995/2012 et, ci-dessous, chap. 10). 